\title{
Décors et costumes: rôles et fonctions dans le roman sadien
}

Didier Derson

\section{Q OpenEdition \\ 1 Journals}

\section{Édition électronique}

URL : https://journals.openedition.org/studifrancesi/25868

DOI : 10.4000/studifrancesi.25868

ISSN : 2421-5856

Éditeur

Rosenberg \& Sellier

\section{Édition imprimée}

Date de publication : 1 avril 2007

Pagination : 59-76

ISSN : 0039-2944

\section{Référence électronique}

Didier Derson, « Décors et costumes: rôles et fonctions dans le roman sadien », Studi Francesi [En ligne], 151 (LI | I) | 2007, mis en ligne le 30 novembre 2015, consulté le 23 novembre 2021. URL : http:// journals.openedition.org/studifrancesi/25868; DOI : https://doi.org/10.4000/studifrancesi.25868

\section{(c)}

Studi Francesi è distribuita con Licenza Creative Commons Attribuzione - Non commerciale - Non opere derivate 4.0 Internazionale. 


\section{Décors et costumes: rôles et fonctions dans le roman sadien}

S'intéresser aux différents problèmes de mise en scène dans l'ensemble des Euvres $^{1}$ du marquis de Sade ne consiste pas uniquement à jeter un regard critique sur la scénarisation de l'espace et l'influence que peuvent avoir certaines esthétiques théâtrales sur ces romans. Le souci de la mise en scène pose également le problème des décors intérieurs et des costumes. Et Maurice Lever ne dit pas autre chose dans l'introduction à l'ouvrage qu'il a consacré aux écrits politiques du divin marquis:

Paradoxalement, c'est l'érotisme, et lui seul, qui parvient à susciter chez lui ce sens de l'ordre, qu'il refoule si énergiquement partout ailleurs. Comment s'en étonner, d'ailleurs, lorsqu'on connaît son irrésistible penchant à théâtraliser la vie sexuelle? Dès lors que la partie de débauche se mue en cérémonial, rien ne saurait être laissé au hasard: ni le choix du décor, ni celui des partenaires, ni le style des dialogues. Il n'est pas jusqu'aux costumes, aux accessoires, aux gestes et aux postures des «acteurs», qui ne fassent l'objet d'une minutieuse mise au point.[...]. Metteur en scène de la transgression érotique, Sade dispose, les groupes, compose les scènes en tableaux vivants, varie les attitudes, combine les corps en vue d'étranges chorégraphies, où la jouissance atteint son paroxysme au gré de copulations acrobatiques, souvent improbables, mais toujours infaillibles ${ }^{2}$.

Notre propos dans ces pages portera sur les accessoires qui donnent vie à ces espaces scéniques, les décors dans lesquels évoluent les protagonistes des romans sadiens, ainsi que sur le rôle prépondérant que joue la lumière dans la mise en scène sadienne. Quant aux costumes, nous montrerons que ceux-ci servent très fréquemment à «sérier» les personnages, c'est-à-dire à les répartir dans des groupes que nous pourrons appeler classes en fonctions des besognes qu'ils ont à accomplir auprès de tel ou tel libertin ou bien encore au cours de telle ou telle scène érotique.

\section{Le décor intérieur et les accessoires de décoration.}

Le premier constat que nous pouvons établir à propos des décors intérieurs se fonde sur le caractère particulièrement conventionnel de ceux-ci. Le marquis de Sade, dans ce domaine, n'a rien d'un écrivain «révolutionnaire», si l'on peut dire. Ses romans historiques respectent le cadre et les accessoires de 1'époque médiévale

(1) SADE (D.A.F., marquis de), Euvres Complètes. Éditions mise en place par Annie Le Brun et Jean-Jacques Pauvert. Paris, Éditions Jean-Jacques Pauvert, 1986-1991, 15 tt.

$N$. B. Les citations que nous empruntons aux œuvres romanesques et théâtrales de Sade renvoient à cette édition. Pour des raisons de commodité et de clarté de l'apparat critique, nous nous contenterons de ne mentionner que le titre du roman ou de l'œuvre théâtrale, la tomaison, et la pagination de la citation.

(2) SADE (D.A.F., marquis de): «Que suis-je à présent...». Sade et la Révolution. Textes politiques choisis, présentés et annotés par Maurice Lever. Paris, Bartillat, 1998, p. 7. 
pour l'Histoire secrète d 'Isabelle de Bavière... et d'Adélaïde de Brunswick... ${ }^{4}$; ceux de l'époque de «la minorité de Louis XIV» pour La Marquise de Gange quoique le goût pour le Moyen Age n'ait pas totalement disparu'.

Pour l'ensemble des autres romans, les décors intérieurs respectent assez scrupuleusement le cadre conventionnel des intérieurs du XVIII ${ }^{\text {eme }}$ siècle à l'instar de ce que l'on rencontre dans bon nombre d'œuvres dramatiques, de Marivaux à Beaumarchais en passant par celles du divin marquis lui-même, sans oublier les œuvres du théâtre érotique de l'époque ${ }^{7}$, par exemple.

En règle générale, nous constatons que le décor demeure relativement vague et imprécis. Ce minimalisme du décor, proche d'une certaine manière de l'abstraction, participe d'une stratégie romanesque qui consiste à octroyer au corps dans tous ses états la place prépondérante qui lui est due, à l'instar de ce que préconisera au début du Xx ${ }^{\text {ème }}$ siècle Adolphe Appia ${ }^{8}$. Peu importe, à la limite, la pauvreté des décors intérieurs. Les enjeux du roman sadien ne sont pas ceux du roman balzacien. Le lecteurspectateur ne doit surtout pas se perdre dans moult détails décoratifs. Son attention toute entière et son regard ne doivent se focaliser que sur des corps en action.

En outre, ce décor intérieur est toujours clos sur lui-même, égocentrique, comme l'est le monde libertin, et dépourvu de fenêtres. Cette singularité a pour conséquence que toute fuite vers l'extérieur, c'est-à-dire vers ce qui appartient encore au monde de l'humanité, ne fut-ce que par le regard, est impossible. Les libertins et leurs victimes sont contraints de s'affronter, dans un monde sans soleil, sans lumière naturelle?. Dans les nombreux huis-clos qui les opposent, le regard devient une arme à double tranchant. Arme destructrice pour le libertin envers sa victime; arme de résistance désespérée pour celle-ci face à celui-là. Toutefois, il existe des exceptions. Dans Justine..., le dénouement de l'action se passe dans un salon muni de fenêtres ${ }^{10}$. Mais ces fenêtres ne permettent pas à notre malheureuse hérö̈ne de s'évader même par le regard, dans la mesure où c'est par cette fenêtre qu'«un éclat de foudre» parviendra à «renverser» Justine, la rejetant «au milieu du salon», c'est-à-dire au cœur même du monde libertin et égocentrique, cause de tous ses maux. Et dans l'Histoire de Juliette..., Dorval jette les «hardes» de ses victimes, après les avoir mises à nu, «par une fenêtre» ${ }^{11}$. Dans ce cas également, la fenêtre ne permet pas de fuir ce monde de débauche. D'ailleurs, comment ces victimes le pourraient-elles, dans un tel état de nudité!

Le marquis de Sade ne semble donc pas attacher, comme nous l'avons dit plus haut, une importance capitale au décor intérieur, de sorte que, très fréquemment, celui-ci ne contient que quelques accessoires qui ont la particularité d'appartenir, de

(3) Histoire secrète d'Isabelle de Bavière, reine de France, t. 12, p. 201.

(4) Adélaïde de Brunswick, princesse de Saxe, t. 12, p. 273.

(5) La Marquise de Gange, t. 11, p. 179.

(6) Ibid., pp. 183-184.

(7) Cf. La Comtesse d'Olonne, p. 100; L'Esprit des Moeurs..., p. 293.; in Théâtre érotique français au XVIII eme siècle, Paris, Jean-Jacques Pauvert au Terrain Vague, 1993

(8) D'après J. Duvignaud et A. Veinstein, Appia accorde une grande importance au corps de l'acteur dans l'espace scénique qu'il occupe. C'est pourquoi, nos deux critiques rappellent le contenu des théories d'Appia en déclarant que: «Certains principes permanents, liés aux qualités propres au corps de l'acteur - en particulier le fait qu'il ait trois dimensions, que ses lignes soient perpendiculaires -, impliquent, pour le mettre en valeur, une certaine architecture scénique. Ce qui doit prédominer, c'est la construction, qui mettra le corps de l'acteur en valeur, et non le décor peint, qui passe au second pian.», in Le Théâtre. Paris, Larousse, «Encyclopoche Larousse», 1976, p. 49.

(9) D'ailleurs n'est-ce pas le souhait utopique de Curval lorsqu'il affirme à ses compagnons de débauche: «Combien de fois, sacredieu, n'ai-je pas désiré qu'on pût attaquer le soleil, en priver l'univers...», Les Cent vingt journées de Sodome, t. 1 , p. 183 .

(10) Justine ou les Malheurs de la vertu, t. 3, p. 311.

(11) Histoire de Juliette ou les Prospérités du vice, t. 8, p. 166. 
près ou de loin, à une même catégorie, celle des accessoires de repos: fauteuil, sofa, canapé, ottomane, voire lit quoique cet accessoire, paradoxalement, soit assez peu usité dans les romans du pensionnaire de la Bastille. En outre, le relevé que nous avons opéré de ces pièces de mobilier nous a permis de les regrouper en deux classes distinctes dont la première serait composée uniquement du fauteuil et la seconde des autres éléments.

Ce classement résulte d'un constat: le fauteuil est le symbole de la toute-puissance du personnage libertin, car installé dans celui-là, il impose son pouvoir aux autres protagonistes qui l'entourent et tout échange avec autrui devient alors impossible; tandis que les autres pièces de mobilier, en revanche, permettent d'établir une forme de communication, aussi minime soit-elle. Car, à l'inverse du fauteuil, -symbole de la singularité-, le sofa, le canapé, l'ottomane et le lit sous-entendent la pluralité.

Le fauteuil, comme nous venons de le signaler, est très souvent l'accessoire de prédilection du libertin. C'est depuis celui-ci qu'il peut, à sa guise, contempler les parties charnelles des victimes qu'il va molester. Dans Justine ou les Malbeurs de la vertu, Severino «attire avec impudence», «vers le fauteuil sur lequel il est assis», la «petite Agnès» afin de procéder à l'examen de ses charmes ${ }^{12}$, et, dans La Nouvelle Justine..., Verneuil reçoit les hommages de ses victimes assis sur «une espèce de trône», autrement dit une variante du fauteuil:

Tout le monde applaudit; et Verneuil, revêtu de l'autorité suprême, se place en conséquence dans une espèce de trône, [...]. Aussitôt qu'il y est, les femmes, les filles, les enfants, les garçons et les vieilles viennent humblement lui présenter leurs fesses à baiser, après trois génuflexions préalables ${ }^{13}$.

Le fauteuil est également, pour le roué, au faîte de sa puissance, l'accessoire nécessaire à l'expression de l'autorité aristocratique dont il jouit. C'est après s'être assis «sur un fauteuil» que le président, dans Les Cent vingt journées de Sodome, prononce «une pénitence analogue aux forces et à l'âge du délinquant» ${ }^{14}$; que Dorval, placé «dans un grand fauteuil», dénude «depuis les pieds jusqu'à la tête» ses victimes avant de prononcer leur condamnation à mort ${ }^{15}$. Mais cet élément de mobilier demeure aussi un accessoire indispensable au libertin lors des «dissertations», par exemple, comme nous le prouvent les extraits suivants de La Philosopbie dans le boudoir:

Le calme s'étant un peu rétabli pendant ces dissertations, les femmes, revêtues de leurs simarres, sont à demi-couchées sur le canapé, et Dolmancé auprès d'elles dans un grand fauteuil ${ }^{16}$.

\section{ou d'Aline et Valcour}

- Oh! pour le coup je m'assois, a dit alors le président, en se laissant tomber dans un fauteuil..., oui, je m'assois, Dolbourg va prêcher, et il y a longtemps que je suis curieux du sermon d'un fermier général... ${ }^{17}$

Mais il n'en demeure pas moins vrai que les sofas, les canapés, les ottomanes et les lits sont également très prisés dans l'ensemble des œuvres de Sade. Ils le sont,

(12) Justine ou les Malheurs de la vertu, t. 3, p. 184.

(13) La Nouvelle Justine au les Malheurs de la vertu, t. 7, p. 183.
(15) Histoire de Juliette au les Prospérités du vice, t. 8 , p. 166

(16) La Philosophie dans le boudoir, t. 3, p. 405. Ce passage est en italique dans le roman car il s'agit d'une didascalie.

(17) Aline et Valcour, t. 4, p. 121. 
moins parce qu'ils participent d'un phénomène de mode au XVIII ${ }^{\text {ème }}$ siècle (pour les sofas et les ottomanes, notamment), que par la possibilité qu'ils présentent d'être des éléments d'échange, tant intellectuels que corporels, entre les différents protagonistes d'une même histoire. Lorsque Justine rencontre pour la première fois le comte de Gernande, ce dernier est «couché sur une ottomane, et [a] près de lui deux jeunes gens» très peu vêtus ${ }^{18}$. Clairwil multiplie les prouesses érotiques sur une ottomane, en compagnie de Juliette et d'une «fille de dix-huit ans» ${ }^{19}$, bien avant que la sœur de Justine ne commence le récit de ses aventures, confortablement installée «au fond d'une ottomane $\gg^{20}$; quant à Braschi et Juliette, tous deux, pour assister à une parodie d'office religieux, sont «couchés dans une ottomane élevée sur une estrade de dix pieds de haut, à laquelle on ne parvenait que par des marches recouvertes de superbes tapis de Turquie». En outre, le narrateur nous révèle que «cette estrade formait un théâtre où vingt personnes pouvaient se tenir à l'aise» ${ }^{21}$.

À défaut d'ottomane, nos multiples héros se contenteront d'un canapé. Dans Les Cent vingt journées de Sodome, il est utilisé tantôt par les principaux protagonistes pour écouter les narrations des historiennes ${ }^{22}$, tantôt par de libidineux personnages pour assouvir leurs plaisirs lubriques:

97. il se fait fouetter sur la plante des pieds, sur le vit, les cuisses, pendant qu'étendu sur un canapé, trois femmes montent à cheval sur lui et lui chient dans la bouche ${ }^{23}$.

Ce constat, valable pour L'École du libertinage, est tout à fait applicable à Justine... à La Nouvelle Justine... et l'Histoire de Juliette... ${ }^{24}$. Quant au sofa, variante du canapé, sa place est également prépondérante dans l'ensemble de nos œuvres ${ }^{25}$.

Seul, le lit demeure un élément de mobilier peu employé dans l'ensemble des œuvres. Cela s'explique par le caractère privé qu'il symbolise. Il représente le lieu de l'intimité conjugale qui, par convention sociale, n'est pas soumis aux regards d'autrui. Or, cette idée d'intimité n'est pas de mise dans le monde romanesque sadien puisque tout doit être vu.

Cependant, nous rencontrons quelques occurrences dans lesquelles le lit est très fréquemment lié à l'idée de la mort dans une acception très générale du terme. Ainsi le lit est l'endroit privilégié de l'agonie d'un personnage. On peut en effet supposer, non sans raison, car le substantif lit n'apparaît pas textuellement, que le moribond, au seuil de la mort, repose dans un lit $^{26}$. La marquise de Gange, après l'attentat dont elle a été victime, renonce au voyage qui devait la conduire chez sa mère, à Avignon, et décide de «s'entourer» pour «ces derniers moments de tout ce qui lui restait de plus cher», «dans son lit que l'on environna de fleurs» ${ }^{27}$.

(18) Justine au les Malheurs de la vertu, t. 3, p. 197; La Nouvelle Justine au les Malheurs de la vertu, t. 7 , p. 118 .

(19) Histoire de Juliette au les Prospérités du vice, t. 8 , p. 320 .

(20) La Nouvelle Justine ou les Malheurs de la vertu, t. 7 , p. 406.

(21) Histoire de Juliette ou les Prospérités du vice, t. 9; p. 201.

(22) Les Cent vingt journées de Sodome, t. 1, p. $110 ; 345$.

(23) Ibid., p. 377.

(24) Nous ne citerons que quelques références extraites de ces trois romans tant les occurrences du substantifs «canapé» sont nombreuses. Justine ou les Malheurs de la vertu, t. 3, p. 136; p. 209; p. 211; p. 242; p. 243; p. 244; La Nouvelle Justine ou les Malbeurs de la vertu, t. 6, p. 183; p. 209; p. 275 ; p. $385 ;$ t. 7 , p. 74 ; p. 99 ; p. $145 ;$ p. 170 ; p. 315; p. 406; Histoire de Juliette ou les Prospérités du vice, t. 8 , p. 105 ; p. 148 ; p. 250; p. 317; p. 491; p. 581 ; t. 9 , p. 18 ; p. 62 ; p. 99 ; p. 108 ; p. 223; p. 297; p. 397; p. 489.

(25) Les Cent vingt journées de Sodome, t. 1, p. 137; p. 328; p. 336; La Nouvelle Justine ou les Malbeurs de la vertu, t. 6, p. 183; p. 267; p. 320; p. 362; t. 7, p. 312; Histoire de Juliette ou les Prospérités du vice, t. 8, p. 355; p. 384; p. 435; p. 535; t. 9, p. 31; p. 163 ; p. 213 ; p. 314 ; p. 397 ; p. 486; p. 489.

(26) Dialogue entre un prêtre et un moribond, t. 1, p. 501 .

(27) La Marquise de Gange, t. 11, p. 374. 
Représentations schématiques comparées de la salle des narrations des Cent vingt journées de Sodome et du théâtre antique grec.

Salle des narrations des Cent vingt journées de Sodome.

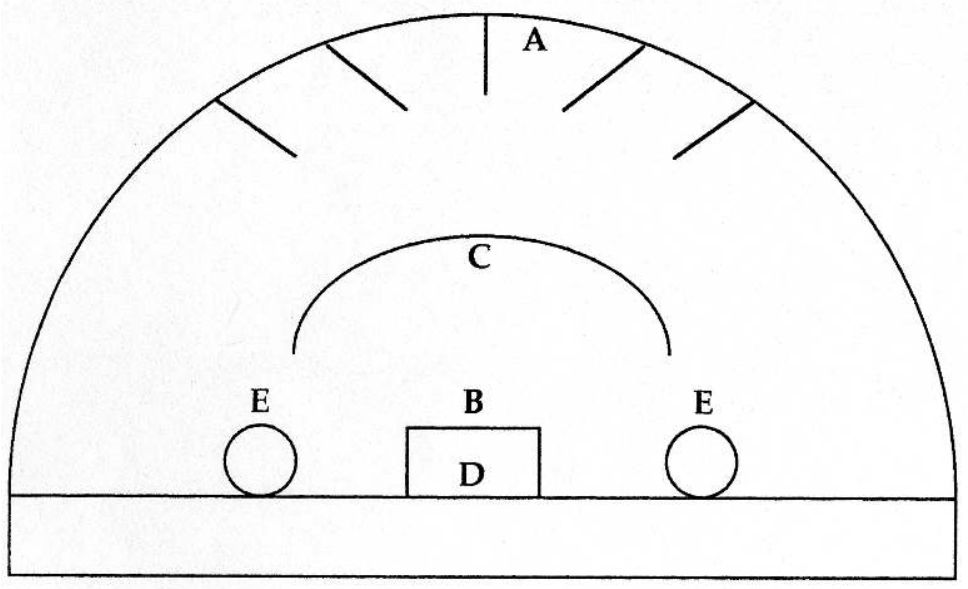

A. Niches ornées de glaces. B. Trône destiné aux historiennes pour leurs narrations. C. Gradins destinés aux victimes des débauches. D. Mur formant le diamètre de la salle des narrations. E. Colonnes isolées destinées à recevoir une victime ayant commis une faute.

Théâtre antique grec.

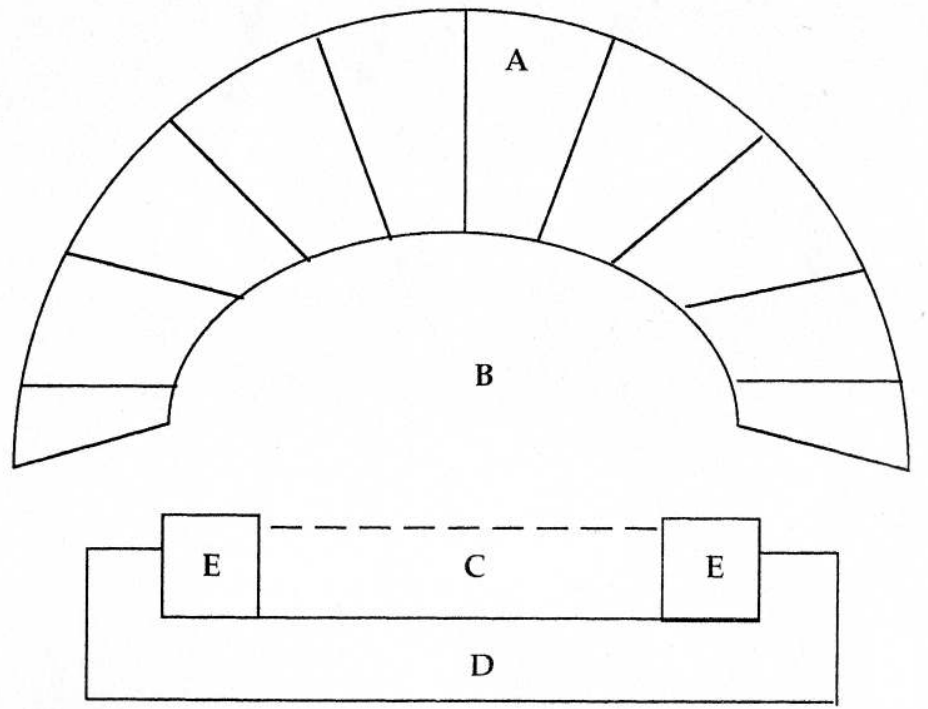

A. Kôilon: gradins. B. Orkhestra: espace circulaire au centre duquel se dressait 1'autel de Dionysos et où évoluaient les chœurs. C. Proskénion: devant de la scène où se tenaient les acteurs. D. Mur de scène. E. Paraskenia: saillies murales fermant à droite et à gauche la scène. 
Le lit peut être également le lieu de la maladie et de la souffrance gratuite. Le repaire de Minski dans Les Prospérités du vice, présente une salle occupée par «vingtcinq lits» qui servent aux «femmes blessées par les intempérances» de l'ermite ou pour «celles qui [tombent] malades» ${ }^{28}$. C'est «sur un lit» que Juliette est sur le point de contracter la petite vérole ${ }^{29}$. Par bonheur, elle se rendra compte de l'état de santé du personnage avec lequel elle devait se livrer à une partie et substituera à sa place une «petite novice de treize ans» ${ }^{30}$. Dans La Nouvelle Justine..., c'est étendu «sur un lit» que Lafleur, le valet de Saint-Florent, attire «Justine sur lui» afin de présenter «le cul de [cette] aventurière aux attentats» de son maître. Dès lors, notre malheureuse héroïne subira des souffrances intolérables qui résulteront de piqûres dispensées à l'aide d'une «longue aiguille d'acier» ${ }^{31}$.

Le lit demeure enfin le lieu de la mort feinte, plus précisément de la petite mort, celle que les personnages éprouvent au plus fort de l'extase érotique. Dans l'Histoire de Juliette..., certaines tribades au service de Delbène, sur le conseil de cette dernière, goûteront «le plaisir qui [leur] conviendra le mieux» dans les bras de Juliette étendue sur un lit ${ }^{32}$. Dans La Nouvelle Justine..., cette mort feinte, lors des jeux érotiques, n'est pas une simple vue de l'esprit. Elle correspond à une volonté du narrateur, puisque la scène dans laquelle se pâment Séraphine et son frère dure, en effet, «près d'une heure» et que l'immobilisme le plus total, symbolisant la mort, y est présent:

Et le fripon, en disant cela, me débarrasse de mes jupons, quitte ses culottes, et, m'ayant couchée sur le lit, il [le frère de Séraphine] s'étend, en sens contraire, sur moi, de manière à pouvoir placer son vit dans ma bouche, et que ses lèvres se posent sur mon con. Je [Séraphine] le suce, il me le rend; nous restons ainsi, près d'une heure, à nous pâmer, sans varier la posture ${ }^{33}$.

Nous avons trouvé également et très épisodiquement d'autres types de sièges. Dans La Nouvelle Justine..., un épisode se déroule «sur un banc étroit» ${ }^{34}$; alors qu'un autre épisode nous présente «un vaste fauteuil à cinq places, construit de manière que ceux qui y sont placés se trouvent dos à dos» ${ }^{35}$; dans un autre encore, la comtesse de Gernande est allongée «dans une chaise longue» ${ }^{36}$; et Justine, soucieuse du respect de l'étiquette prend place sur une «chaise» lorsque sa sœur commence d'entreprendre le récit de ses aventures ${ }^{37}$. Enfin, dans Les Prospérités du vice, Delcour «renverse» Juliette «sur une large bergère ${ }^{38}$,tandis que, bien plus tard, chez Sophie de Prusse, notre Messaline sera pieds et poings liés sur une «banquette» ${ }^{39}$.

$$
* * *
$$

Le marquis de Sade peut, dans quelques cas précis, présenter à son lecteurspectateur des décors intérieurs d'une assez grande précision. Dans ce cas de figure, nous constatons que ceux-ci renvoient immanquablement au monde du spectacle, ou si l'on préfère au monde du théâtre. Parce que, dans ce type de décor, tout est

(28) Histoire de Juliette ou les Prospérités du vice, t. 9 , p. 10 .

(29) Ibid., t. 8, p. 173.

(30) Ibid., p. 174.

(31) La Nouvelle Justine ou les Malbeurs de la vertu, t. 7, p. 251.

(32) Histoire de Juliette ou les Prospérités du vice, t. 8 p. 72 .

(33) La Nouvelle Justine ou les Malbeurs de la vertu, t. 7 , p. 276.

(34) Ibid., t. 6, p. 348.
(35) La Nouvelle Justine ou les Malbeurs de la vertu, t. 7 , p. 202.

(36) Ibid. p. 145. Nous retrouvons sensiblement la même scène dans Justine ou les Malheurs de la vertu, t. 3, p. 210.

(37) La Nouvelle Justine ou les Malheurs de la vertu, t. 7 p. 406.

(38) Histoire de Juliette ou les Prospérités du vice, t. 8, p. 332

(39) Ibid., t mef 9, p. 228. 
conçu pour que les protagonistes de l'histoire puissent voir et être vus et que le lecteur devienne bien davantage un spectateur. C'est pourquoi ces décors prennent la forme de scènes théâtrales ou bien encore la forme d'un amphithéâtre dont l'architecture rappelle celle du théâtre antique; et que bien souvent les niches et les alcôves se parent d'une multitude de glaces.

Le plus bel exemple de décor intérieur qui rappelle la scène théâtrale où s'exhibent les acteurs est sans conteste celui que le narrateur des Cent vingt journées de Sodome ${ }^{40}$ donne à découvrir à son lecteur:

Il [le salon des narrations] était d'une forme demi-circulaire. Dans la partie cintrée se trouvaient quatre niches de glaces fort vastes et ornées chacune d'une excellente ottomane; ces quatre niches, par leur construction, faisaient absolument face au diamètre qui coupait le cercle. Un trône élevé de quatre pieds était adossé au mur formant le diamètre. Il était pour l'historienne: position qui la plaçait non seulement bien en face des quatre niches destinées à ses auditeurs, mais qui même, vu que le cercle était petit, ne l'éloignant point trop d'eux, les mettait à même de ne pas perdre un mot de sa narration, car elle se trouvait alors placée comme est l'acteur sur un théâtre, et les auditeurs, placés dans les niches, se trouvaient l'être comme on l'est à l'amphithéâtre. Au bas du trône étaient des gradins sur lesquels devaient se trouver les sujets de débauche amenés pour servir à calmer l'irritation des sens produite par les récits: ces gradins, ainsi que le trône, étaient recouverts de tapis de velours noir garnis de franges d'or, et les niches étaient meublées d'une étoffe pareille et également enrichie, mais de couleur bleu foncé. [...] Des deux côtés du trône, il y avait une colonne isolée et qui allait toucher le plafond; ces deux colonnes étaient destinées à contenir le sujet que quelque faute aurait mis dans le cas d'une correction. Tous les instruments nécessaires à cette correction étaient accrochés à la colonne $[\ldots]^{41}$.

(40) Voici ce que déclare Michel Tort dans son article intitulé «l'Effet Sade», Tel Ouel, $\mathrm{n}^{\circ} 28$, Paris, hiver 1967, p. 77, à propos de la salle des narrations des Cent vingt journées de Sodome: «Ce lieu [la salle des narrations] est un théâtre d'un modèle et d'une utilisation assurément inédite». «De forme semicirculaire», il se compose de deux parties. La partie centrée s'en divise en quatre niches de glaces abritant les pervers auditeurs-spectateurs auxquels fait face sur la scène un personnage vicariant, la conteuse qui récite le dict pervers, compromis entre le tableau et le récit. «Elle se trouvait alors placée comme l'est l'acteur sur un théâtre et les auditeurs placés dans les niches se trouvaient l'être comme on l'est à l'amphithéâtre». Mais à vrai dire, du théâtre, celui-là n'est que la subtile perversion, comme il va se voir, le lieu de la plus énorme dérision de la catharsis. D'abord par une discrète inversion des rôles respectifs de l'acteur et du spectateur. La participation de l'acteur unique s'y réduit en effet au dévidement linéaire de sa parole, par l'organe de laquelle se déroule le tableau de la perversion. $\mathrm{n}$ n'est que le fragile support de ce tableau, comme le montre suffisamment sa disparition dans les esquisses des dernières parties, qui ne présentent que les schémas d'actes pervers dissociés de tout récit. Réduit à être le lieu de l'énonciation du tableau, l'acteur n'intervient en fait qu'en vertu d'une analogie entre cette représentation qu'est le tableau et la représentation théâtrale. Du coté du spectateur, la situation n'est pas moins curieuse: l'acte, dissocié de la parole, disparu de la scène, se retrouve là comme effet de la pure représentation dans le lan- gage. Mais il y faut une médiation très particulière qui achève de signer la restructuration perverse de l'espace théâtral. Car le regard, n'ayant rien à percevoir sur la scène se dédommage en se reportant au-dessous: «au bas du trône étaient des gradins sur lesquels devaient se trouver les sujets de débauche amenés pour servir à calmer l'irritation des sens produite par les récits». Par ce déplacement des gradins projetés ainsi en avant du lieu du spectacle, les pervers à mesure dessinent, ébauchent sur les corps des victimes qui leur font face la réalisation de la représentation, dans ce lieu intermédiaire entre le réel et l'imaginaire, entre la salle et la scène.

Tout étant ainsi disposé pour la mise en ceuvre de la représentation, l'effet Sade, à la production duquel se réduit exactement la pièce, peut s'accomplir. Alors, en chaque point du récit des conteuses, un des protagonistes se trouvera pris, et, reconnaissant sa marque, selon la loi énoncée plus haut, ira choir hors de sa place. Ce mouvement par lequel les sujets, frappés par le signifiant, à tour de rôle, quittent la scène pour se retirer, en arrière, dans des pièces prévues à cet effet, ne fait que matérialiser l'effet de clivage et d'effacement du sujet suscité par la représentation. Personnages du roman et lecteurs sont bien soumis, dans le fantasme de Sade, à l'efficace de la même structure; le théâtre que se donnent les pervers n'est que la représentation concrète, le redoublement dans un scénario visible des effets escomptés du tableau par là même représenté sur le lecteur». p. 64. 
Dans ce salon, le divin marquis s'est assurément souvenu de l'architecture du théâtre antique ${ }^{42}$. En effet, «les niches» où s'installent Durcet, Curval, Blangis et son frère l'Évêque rappellent les gradins (kôilon) ou prennent place les spectateurs. «Le trône élevé de quatre pieds» nécessaire aux historiennes pour leurs narrations évoque le proskênion, c'est-à-dire la scène sur laquelle évoluent les acteurs; de plus, ce «trône» est «élevé» exactement comme l'est la scène du théâtre grec.

Les «gradins» au pied de la tribune des narrations ne sont pas sans relation avec l'orkhêstra où se dressait l'autel de Dionysos autour duquel évoluait le chœur antique. Et les deux «colonnes» aux accessoires symbolisent, davantage par leur position que par le rôle qu'elles jouent dans ce cabinet, les deux parois, - les paraskénia -, qui fermaient de part et d'autre le proskênion. Enfin ce salon en forme d'hémicycle est fermé par un mur épousant le diamètre, à l'instar du mur extérieur formant façade du théâtre antique grec ou romain. On rencontre également, dans l'Histoire de Juliette..., un autre exemple d'espace scénique qui épouse la forme semi-circulaire du théâtre antique. Il s'agit d'un «vaste kiosque» qui abrite dans «la partie du fond»

un amphithéâtre demi-circulaire, s'élevant à trois pieds du sol, garni d'épais matelas recouverts de satin couleur feu, sur lesquels on pouvait se coucher à l'aise; en face était un gradin, plus haut d'un pied, d'égale forme, qu'un vaste tapis de velours de même couleur garnissait en entier ${ }^{43}$.

Cette volonté du marquis de Sade de transformer chaque espace de débauche en une scène hémisphérique n'est pas le résultat d'un goût prononcé pour l'antiquité. Cette forme architecturale est souhaitée parce qu'elle permet, comme nous l'avons déjà dit par ailleurs, de voir et d'être vu; d'être, pour nos multiples protagonistes, tout à la fois spectateurs et acteurs de leurs propres débauches. Et cela n'est possible que par la volonté de confondre l'espace scénique en tant que tel, c'est-à-dire le lieu de la représentation théâtrale, avec ce que Donatien appelle «les gradins», c'est-à-dire l'espace réservé aux spectateurs ${ }^{44}$.

D'une certaine manière, cette profonde volonté de confondre dans un même espace acteur et spectateur pose le problème de la notion même de représentation théâtrale. Car qu'est-ce qu'une représentation théâtrale sinon une sorte de pacte tacite, voire inconscient, entre des acteurs qui, installés sur une scène, interprètent une œuvre dramatique et un public qui assiste à sa représentation. Or, Donatien, dans une grande partie de son œuvre romanesque, remet en cause, comme nous venons de le voir, cette tradition; de sorte que nous pouvons nous demander si, par un excès de théâtralité, le divin marquis n'en arrive pas à créer une sorte d'antithéâtre, peut-être une forme de happening bien avant l'heure? Cette idée demeure sous-jacente dans les propos d'Annie Le Brun, quoiqu'elle ne parle pas d'antithéâtre, lorsqu'elle évoque «la démolition théâtrale» entreprise par le marquis de Sade dans Les Cent vingt journées de Sodome ${ }^{45}$.

(42) Voir schéma page suivante.

(43) Histoire de Juliette ou les Prospérités du vice, t. 9 , p. 374 .

(44) Par cette réalité du monde romanesque sadien, nous pouvons affirmer que le divin marquis applique, inconsciemment peut-être, une conception des rapports scène-salle que théorisera quelque deux siècles plus tard Antonin Artaud dans son Premier manifeste du Théâtre de la Cruauté, p. 148: «Nous supprimons la scène et la salle qui sont rem- placées par une sorte de lieu unique, sans cloisonnement, ni barrière d'aucune sorte et qui deviendra le théatre même de l'action. Une communication directe sera rétablie entre le spectateur et le spectacle, entre l'acteur et le spectateur, du fait que le spectateur placé au milieu de l'action est enveloppé et sillonné par elle. Cet enveloppement provient de la configuration même de la salle».

(45) Voici ce que rappelle Annie Le Brun, à propos des Cent vingt journées de Sodome, dans L'ina- 
Le narcissisme et l'égocentrisme du libertin n'ont pas de limite. Voir et être vu demeurent ses principales préoccupations, et toutes les parties du corps doivent offrir la possibilité d'être disséquées du regard quels que soient les points de vue. Aussi n'est-il pas surprenant que nous rencontrions un nombre important de «glaces» dans les appartements de nos héros libertins. Car, comme le souligne Jean-Marie Goulemot dans son essai, toute la stratégie du roman érotique ou pornographique «se fonde sur le regard $»^{46}$. Mais le théâtre érotique n'est pas en reste non plus, puisque l'action dramatique de L'Esprit des Mceurs au XVIII' siè siècle ou La Petite maison de Bernard de Saint-Just (1789) se déroule, pour le premier acte, dans «un salon très-orné (sic) de glaces....» ${ }^{47}$. En outre, la présence de ces miroirs qui, dans bien des exemples que nous citerons, se multiplient «à l'infini», participe de la volonté du marquis de Sade de rendre protéiforme toute la gestuelle amoureuse des héros afin de procurer, chez le lecteur-spectateur qui ne participe pas charnellement aux ébats, un plaisir profond, décuplé par le désir qui naît de cette perception multiple. Dans La Philosophie dans le boudoir, Eugénie s'extasie devant une «délicieuse niche» et interroge madame de Saint-Ange sur la présence d'une multitude de miroirs: «Mais pourquoi toutes ces glaces?». Et cette dernière de répondre avec empressement que:

C'est pour que, répétant les attitudes en mille sens divers, elles multiplient à l'infini les mêmes jouissances aux yeux de ceux qui les goûtent sur cette ottomane. Aucune des parties de l'un et l'autre corps ne peut être cachée par ce moyen: il faut que tout soit en vue; ce sont autant de groupes rassemblés autour de ceux que l'amour enchaîne, autant d'imitateurs de leurs plaisirs, autant de tableaux délicieux, dont leur lubricité s'enivre et qui servent bientôt à la contempler elle-même ${ }^{48}$.

Dans La Nouvelle Justine..., Abraham Pexoto, un des nombreux roués que Justine croise sur sa route, souhaite que:

deux jolies filles, qu'il avait attachées au service de Joséphine, la branlassent sous ses yeux dans un boudoir de glaces, en lui faisant prendre pendant la séance huit ou dix attitudes différentes ${ }^{49}$.

Et les domestiques au service du comte de Gernande préparent «le plus beau salon du château» dans le fond duquel est placée «une large ottomane» qui était entourée de «tant de glaces qu'il devenait impossible que les scènes qu'on allait exécuter dans ce superbe local ne s'y multipliassent pas sous mille et mille formes $\gg^{50}$. Enfin, dans l'Histoire de Juliette..., le lecteur-spectateur découvre que les principaux héros libertins du roman possèdent tous un salon, un boudoir, voire un cabinet orné de glaces. Au fond de la chambre de l'innommable Minski, nous découvrons «une

nité de la littérature, p. 100: «Faut-il s'étonner alors qu'afin de mener à bien cette démolition théâtrale de tous les ressorts de la comédie humaine, Sade subvertisse complètement l'idée de théâtre comme lieu public pour dresser le théâtre le plus secret qu'on puisse concevoir «dans un pays sur, au fond d'une forêt inhabitable, dans un réduit de cette foret que, par les mesures prises, les seuls oiseaux du ciel pouvaient aborder? et c'est pourquoi Les Cent vingt journées peuvent être considérées comme la vision saisissante d'un théâtre enfin débarrassé de ses implications sociales. En cela réside leur puissance critique faisant de la scène un lieu absolument intérieur mais aussi le seul lieu d'où voir de l'intérieur...»

(46) J. -M. Goulemot, Ces Livres qu'on ne lit que d'une main. Lecture et lecteurs de livres pornographiques au XVIII siècle. Paris, Alinéa, 1991, p. 55.

(47) L'Esprit des Mours au XVIII ${ }^{\text {ime }}$, siècle ou La Petite maison, in Op. cit., p. 293.

(48) La Philosophie danse boudoir, t. 3, p. 399.

(49) La Nouvelle Justine ou les Malheurs de la vertu, t. 6, p. 398.

(50) Ibid., t. 7, p. 180. 
vaste alcôve entourée de glaces ${ }^{51}$; la duchesse de Grillo, dans la quatrième partie du roman, fait entrer Juliette et ses amis dans «une salle ronde» où

des glaces, multipliées à l'infini, achevaient de rendre ce petit local l'un des plus jolis temples que Vénus eût en Italie ${ }^{52}$.

Juliette, dans l'avant dernière partie des Prospérités du vice a rendez-vous avec l'impératrice Catherine de Russie dans une «maison de campagne», plus précisément «au sein d'un boudoir» dans lequel le mobilier est environné «de glaces qui se voyaient au-dessus» ${ }^{53}$ et poursuivra ses obscénités avec le roi de Naples et une jeune femme dénudée «sur un canapé, dans une niche de glaces» ${ }^{54}$.

La lumière, enfin, joue un rôle prépondérant dans les décors intérieurs. Très fréquemment celle-ci est peu importante, ce qui donne souvent naissance à une obscurité plus ou moins dense qui n'est pas sans rappeler la technique du clair-obscur employée en peinture. Dans La Marquise de Gange, Euphrasie trouve souvent refuge dans «la chapelle du château» qui est «simplement éclairé[e] par une coupole» ${ }^{55}$ et Léonore, dans Aline et Valcour, est conduite, par «un corridor si sombre qu'à peine y voyait-on», dans «une haute et grande salle voûtée, $[\ldots]$, uniquement éclairée par des lampes ${ }^{56}$.

Toutefois, aussi passionnant que puisse être cet aspect, nous ne nous y attacherons pas de davantage, car il nous semble que la présence de la pleine lumière, de la lumière vive dans les décors intérieurs offre un plus grand intérêt. Il n'est pas rare, en effet, de découvrir des appartements qui baignent dans une lumière vive, voire éblouissante. Ce souci de Donatien n'est pas un simple caprice d'auteur, mais participe bien d'une stratégie de mise en scène au sein de laquelle le rôle de la lumière participe d'une profonde volonté, tout comme les glaces d'ailleurs, de mettre en avant, pour ne pas dire en lumière, le ballet protéiforme et érotique des corps. Car la lumière dans le monde romanesque du divin marquis n'est pas tant au service du décor qu'à celui des corps en action. Or cette conception est très novatrice, voire avantgardiste pour l'époque, dans la mesure où elle ne sera reprise et mise en forme que par Adolphe Appia au début du Xx ${ }^{\text {ème }}$ siècle. Ainsi, messieurs Duvignaud et Veinstein, dans leur petit ouvrage consacré au théâtre, insistent sur l'«importance considérable accordée par Appia à la lumière, qui doit être utilisée en vue non plus d'éclairer le décor, mais de mettre en valeur les qualités plastiques du corps de l'acteur» ${ }^{57}$.

Dans Justine ou les Malheurs de la vertu, notre hérö̈ne est conduite par Severino dans «une salle charmante et magnifiquement éclairée» au point que Justine en est éblouie:

Un escalier se présente; au bout de trente ou quarante marches, une porte s'ouvre, des reflets de lumière viennent frapper mes yeux... ${ }^{58}$

Mais la stupeur de cette dernière ne s'arrête pas là, car le «spectacle qu'elle découvre» met bien en scène les corps de certains personnages:

(51) Histoire de Juliette ou les Prospérités du vice, t. 8 , p. 604 .

(52) Ibid., t. 9 , p. 62.

(53) Ibid., t. 9 , p. 278

(54) Ibid., p. 336.
(55) La Marquise de Gange, t. 11, p. 188.

(56) Aline et Valcour, t. 5, pp. 205-206.

(57) J. Duvignaud et A. Veinstein, op. cit., p. 50. p. 131 
...nous entrons dans une salle charmante et magnifiquement éclairée; là je vois trois moines et quatre filles autour d'une table servie par quatre autres femmes toutes nues ${ }^{59}$.

et dans la troisième version des Infortunes de la vertu, elle fait connaissance «de l'évêque de Grenoble», «à demi couché sur une ottomane», «dans un appartement aussi somptueux que bien éclairé» ${ }^{60}$; quant à Olympe et Juliette, après s'être livrées au libertinage le plus effréné, elles s'apprêtent «à se mettre à table» dans «un cabinet aussi voluptueusement meublé que magnifiquement éclairé» ${ }^{61}$.

Parfois, Donatien insiste sur l'intensité lumineuse qu'il donne à voir aux protagonistes des romans et au lecteur-spectateur. Ainsi, dans l'Histoire de Juliette..., notre Messaline est invitée par Minski à une bacchanale dans une «salle» dont la lumière provient de «vingt-quatre têtes de morts [qui] renfermaient entre elles une lampe dont les rayons sortaient par les yeux et les mâchoires $»^{62}$; Noirceuil se prépare à une orgie qui se tiendra dans une «salle» éclairée de «cent bougies» ${ }^{63}$. Dans ces deux exemples, la quantité de lumière conserve des proportions tout à fait raisonnable. Mais ce n'est pas toujours le cas, et certaines quantités mentionnées par le divin marquis demeurent démesurées et invraisemblables. Cela est particulièrement vrai dans l'Histoire de Juliette... Dans la troisième partie de ce roman, notre Lucrèce est conviée à un repas fastueux dans une salle où la lumière est omniprésente pour ne pas dire omnipotente, puisque le narrateur affirme que «des millions de lumières» éclairaient cette pièce ${ }^{64}$. D'une telle puissance lumineuse, il serait logique que le lecteur-spectateur s'attendît à ce qu'il y eût un formidable éblouissement ou qu'il fût le témoin d'une situation quasiment insoutenable tant par la puissance du rayonnement que par la chaleur qui naît inévitablement de ces sources lumineuses. Or, il n'en est rien, - et c'est bien sur cette incohérence que repose toute l'invraisemblance de cet épisode et toute la portée ironique qui parcourt également l'ensemble du monde romanesque sadien -, car ces «millions de lumières» ne diffusent que «la clarté la plus douce»! Dans un autre épisode, le divin marquis emploie une métaphore, -qui n'est pas sans rappeler les idées de fluidité et de mouvement chères au monde baroque-, où la source lumineuse équivaut à «des flots abondants de lumières»; mais ici aussi l'invraisemblance est encore de mise, car ces «flots abondants», qui sont censés mettre sur le devant de la scène toute la «plastique des corps» de nos lubriques héros, sont simplement émis par des «lampes nourries d'huile de jasmin»:

Tout agissait; tout bandait; tout se prêtait. On n'entendait que des cris ou de plaisir ou de douleur, et le murmure délicieux des cinglons de verges. tout était nu; tout présentait la lubricité sous ses faces les plus scandaleuses. Des flots abondants de lumière, produits par les lampes nourries d'huile de jasmin, dont le parfum flattait autant l'odorat que les rayons enchantaient les yeux, achevaient de rendre ce temple l'un des asiles les plus délicieux que la luxure eut encore vus s'ériger pour elle ${ }^{65}$.

Enfin, l'intensité artificielle de la lumière, nécessaire au bon déroulement d'une scène orgiaque, peut atteindre la puissance de la lumière solaire et devenir ainsi un important adjuvant à nos lubriques héros. C'est ce que nous avons constaté dans un épisode, riche en termes théâtraux, de l'ultime partie des Prospérités du vice:

(59) Ibid., p. 131.

(60) La Nouvelle Justine ou les Malheurs de la vertu, t. 7, p. 344.

(61) Histoire de Juliette ou les Prospérités du vice, t. 9 , p. 71 .
(62) Histoire de Juliette ou les Prospérités du vice, t. 8, p. 603 .
(63) Ibid., t. 9, p. 568.
(64) Ibid., t. 8, p. 461.
(65) Ibid., t. 9, p. 516. 
Un faisceau énorme de lumière partant du plafond répandait dans cette salle une flamme aussi pure que celle du soleil, et avait cela de particulier que les rayons de ce feu ardent, adroitement dirigés sur une infinité d'enfants placés dans le balcon du cintre, les brûlaient au point de leur faire jeter les hauts cris ${ }^{66}$.

Dans ce cas, ce n'est plus précisément l'intensité lumineuse qui est primordiale mais bien davantage la source de chaleur qui résulte de ce rayonnement permettant d'infliger à la victime, devenue le point focal de l'action qui se joue sous nos yeux, les pires souffrances.

\section{Les costumes dans le roman sadien.}

Le marquis de Sade, dans l'ensemble de ses œuvres romanesques, accorde une grande importance aux costumes que portent les multiples protagonistes. Dans ce domaine, nous nous sommes rendu compte que le divin marquis, - à l'inverse de certains aspects du décor intérieur -, n'a rien d'un écrivain novateur. Ce dernier, en effet, fait en sorte que les costumes qui habillent ses personnages soient en phase avec la mode, si l'on peut dire, les goûts et les traditions de l'époque au cours de laquelle se déroule l'action. Dans les premières pages du troisième chapitre d'Adélaïde de Brunswick..., nous apprenons que le prince de Saxe quitte la cour vêtu d'un «simple costume de chevalier» ${ }^{67}$, et que dans l'Histoire secrète d'Isabelle de Bavière..., l'écharpe de couleur qui permet de reconnaître les membres d'une même faction est portée très distinctement:

Enfin, les livrées s'arborèrent. Les Armagnacs se distinguaient par une écharpe blanche, dont un de leur bras était enveloppé. Les Bourguignons prirent la croix de Saint-André, soutenue d'une écharpe rouge ${ }^{68}$.

Toutefois, les costumes sont davantage détaillés dans les romans à caractère pornographique dont les actions, pour la plupart, se déroulent au XVIII ${ }^{\text {ème }}$ siècle. Ce goût du détail est particulièrement sensible dans les soins qu'apporte Donatien pour qualifier telle ou telle étoffe. Dans Justine ou les Malheurs de la vertu, notre malheureuse héroïne est «vêtue d'un mauvais petit caraco d'indienne et enveloppée jusqu'aux sourcils d'un grand mantelet de taffetas noir» ${ }^{69}$; le comte de Gernande, «couché sur une ottomane» est «enveloppé dans une robe de satin des Indes» ${ }^{70}$. Lorsqu'il reçoit la Dubois et Justine, l'évêque de Grenoble, dans La Nouvelle Justine..., est affublé d'une «robe de chambre de taffetas violet»" et le narrateur des Cent vingt journées de Sodome préconise que les fouteurs soient «en gilet et caleçon de taffetas couleur de rose» ${ }^{72}$. Aux côtes des étoffes de taffetas, d'indienne et de satin des Indes, il est possible également de trouver des étoffes de gaze ${ }^{73}$. C'est pourquoi celle-ci est vivement recommandée pour la confection des costumes des «petits garçons» et «des petites filles» des «quatrain» ${ }^{74}$, dans L'École du libertinage. Le goût pour cette matière est

(66) Histoire de Juliette ou les Prospérités du vice, t. 9 ,

(67) Adélaïde de Brunswick, princesse de Saxe, t. 12 , p. 309.

(68) Histoire secrète d'Isabelle de Bavière, reine de France, t. 12, p. 151.

(69) Justine au les Malheurs de la vertu, t. 3, p. 33
(70) Ibid., t. 3, p. 197.

(71) La Nouvelle Justine ou les Malheurs de la vertu, t. 7, p. 344.

(72) Les Cent vingt journées de Sodome, t. 1, p. 71.

(73) Gaze: espèce d'étoffe fort claire, faite de soie ou de fil d'or et d'argent.

(74) Les Cent vingt journées de Sodome, t. 1, p. 71. 
également sensible dans La Nouvelle Justine... Sur l'ordre de Jérôme, Victoire et Justine doivent se rendre dans l'appartement des enfants afin de les costumer de «gazes», de «rubans» et de «fleurs» pour les bacchanales qui s'ensuivront ${ }^{75}$, et bien plus tard dans le déroulement de l'action romanesque, le narrateur invite le lecteur-spectateur aux prémices d'une orgie où le comte de Gernande dénude deux jeunes hommes en lâchant «le nœud coulant d'un gros flot de ruban rose qui retenait des culottes de gaze blanche» ${ }^{76}$; quant à Clairwil, elle invite Juliette à se rendre dans quelques cellules d'un «sérail féminin» où cette dernière découvre des «créatures vraiment célestes» qui «étaient en chemise de gaze» ${ }^{77}$. Enfin, il n'est pas rare de trouver, ici ou là, des pièces de crêpe dont la particularité est d'être surtout une étoffe utilisée lors de séances orgiaques dont l'aspect morbide est très fortement marqué. Dans La Nouvelle Justine..., une victime, «en larmes ou évanouie», des moines libidineux de Sainte-Marie-des-Bois est «revêtue d'un crêpe noir», car, aux dires du narrateur, c'est dans ce «complément barbare» qu'ils puisent les ressources nécessaires «à leur affreux délire ${ }^{78}$. Dans Les Prospérités du vice, Delcour, Juliette et Saint-Fond, avant d'immoler tous les membres de la famille de Cloris, prennent soin de les dénuder et de les «draper d'un crêpe noir» ${ }^{79}$; quant au roi de Naples, ce dernier demande que «quatre jeunes filles de quinze ou seize ans» soient « revêtues de crêpes noirs sous lesquels elles étaient nues» avant d'être suppliciées et enfouies dans «un trou destiné à les recevoir» ${ }^{80}$.

La couleur des étoffes et des costumes semble également un souci important $\mathrm{du}$ divin marquis. Et nous avons pu déjà nous en rendre compte dans les quelques exemples cités précédemment. Cependant, attardons-nous un peu sur cet aspect. Nous constatons que Donatien emploie beaucoup les couleurs rose et bleu, à l'instar de ce qui se pratique dans la peinture baroque française de la deuxième moitié du XVII ${ }^{\text {ème }}$ siècle et de la première du XVIII ${ }^{\text {ème }}$ siècle. Certaines jeunes filles utiles à Braschi pour ses débauches ont «une simarre de taffetas bleu» ${ }^{81}$; Juliette quitte le couvent où elle était pensionnaire, après le décès de ses parents, vêtue d'«une lévite bleue bien en désordre» ${ }^{82}$; les bardaches, au service du comte de Gernande portent des «culottes de gaze blanche» qui sont retenues par des «rubans rose» ${ }^{83}$; dans Aline et Valcour, nous croisons une jeune femme dont les «très beaux cheveux noirs» sont retenus par «un ruban rose ${ }^{84}$. Mais d'autres couleurs viennent enrichir la palette qu'utilise le pensionnaire de Charenton pour peindre les effets vestimentaires de ses héros. Sur l'île d'Otaïti, les habitant de la ville de Tamoé n'utilisent que «le gris, le rose et le vert» pour la confection de «leurs habits» ${ }^{85}$. Et Léonore et Clémentine rencontrent «un burlesque personnage» affublé «d'un pourpoint vert et d'un manteau jaune» ${ }^{86}$. Dans Les Cent vingt journées de Sodome, la palette utilisée par Donatien pour brosser les multiples accoutrements des «jeunes amants» est si riche, si précise et si empreinte de réalisme qu'elle présente à elle seule toutes les couleurs et les étoffes à la mode au cours du XVIII ${ }^{\text {ème }}$ siècle $^{87}$, et semblerait

(75) La Nouvelle Justine ou les Malbeurs de la vertu, t. 6, p. 388.

(76) Ibid., t. 7, p. 125.

(77) Histoire de Juliette ou les Prospérités du vice, t. 8 , p. 464.

(78) La Nouvelle Justine ou les Malheurs de la vertu, t. 6, pp. 306-307.

(79) Histoire de Juliette ou les Prospérités du vice, t. 8 , p. 351 .

(80) Histoire de Juliette ou les Prospérités du vice, t. 9 , p. 397.

(81) Ibid., p. 202.
(82) Justine ou les Malbeurs de la vertu, t. 3, p. 29.

(83) La Nouvelle Justine ou les Malbeurs de la vertu, t. 7, p. 125.

(84) Aline et Valcour, t. 4, p. 178.

(85) Ibid., p. 283.

(86) Aline et Valcour, t. 4, p. 118.

(87) A. LE BRun ajoute la chose suivant à propos de la conception théâtrale des Cent vingt journées de Sodome: «Il s'agit très concrètement d'un théâtre puisque, pour ouvrir là les perspectives illimitées de la passion érotique, Sade n'en part pas moins de 
ainsi être celle utilisée par François Boucher par exemple lorsqu'il peignit, en 1742, La Toilette:

C'était une espèce de petit surtout étroit, leste, dégagé comme un uniforme prussien, mais infiniment plus court et n'allant guère qu'au milieu des cuisses; ce petit surtout, agrafé à la poitrine et aux basques comme tous les uniformes, devait être de satin rose doublé de taffetas blanc, les revers et les parements étaient de satin blanc et, dessous, était une espèce de veste courte ou gilet, également de satin blanc et la culotte de même; [...]. Leurs cheveux, négligemment relevés de quelques boucles sur les côtés, étaient absolument libres et flottants parderrière et simplement noués d'un ruban de la couleur prescrite. Une poudre très parfumée et d'une teinte entre le gris et le rose colorait leur chevelure. [...] Leur tête était nue; un bas de soie blanc à coins brodés de rose couvrait leur jambe qu'un soulier gris, attaché d'un gros nœud rose, chaussait agréablement. Une cravate de gaze à la crème voluptueusement nouée se mariait à un petit jabot de dentelle, et, en les examinant ainsi tous les quatre, on pouvait assurer qu'il ne pouvait, sans doute, rien se voir de plus charmant au monde ${ }^{88}$.

La précision des costumes que nous offre le marquis de Sade dans son monde romanesque n'est en fait que le même travail entrepris par ce dernier dans son œuvre dramatique, et plus précisément dans L'Égarement de l'infortune ${ }^{89}$, Les Antiquaires ${ }^{90}$ ou bien encore dans Henriette et Saint-Clair ou La Force du sang ${ }^{11}$. De sorte que ce constat jette encore une passerelle supplémentaire entre les œuvres romanesques et dramatiques du divin marquis.

Le souci du détail vestimentaire porte également sur les coiffures des personnages. Tantôt elles présentent des cheveux négligemment soignés, tantôt des chevelures strictes et particulièrement bien mises, à l'instar des «deux jeunes gens coiffés» avec beaucoup d'«élégance» et d'«art» qui entourent le comte de Gernande lorsqu'il

dispositifs scéniques et de conventions théâtrales qu'il manie en professionnel et où une historienne du théâtre comme Noëlle Guibert peut relever des éléments de décors et de costumes précis et datables», in De l'inanité en littérature, Paris, Jean-Jacques Pauvert aux Belles Lettres, 1994, pp. 96-97.

(88) Les Cent vingt journées de Sodome, t.1, p. 145.

(89) L'Égarement de l'infortune, t. 1, p. 415: «Costumes.

DERVAL doit être dans le délabrement de la plus grande misère, sans épée, n'ayant de noble que la contenance.

CÉCILE et Marianne sont vêtues fort simplement, il n'y a pas grande différence dans leurs habits; elles peuvent être indifféremment ou en robes usées et fanées à la française, ou en robes un peu plus frầches, mais infiniment plus simples à l'anglaise.

MERVILLE, père, est en pelisse au second acte, c'est une nuit d'hiver; au troisième il vient en velours noir ou brun brodé d'or.

LE CHEVALIER paraît très élégamment mis au premier acte pour produire un plus grand contraste, il vient en frac au trois.

L'EXEMPT et LES RECORS suivent le costume anglais».

(90) Les Antiquaires, t. 14, p. 13: «Costumes.

M. de GIRASOLE, DES COLONNES, et de LEMERAUDE doivent, être vêtus comme le sont ordinairement au théâtre les pères, et les rôles a manteaux, perruques, cannes, habits bruns ou noirs.

AGATHE est avec le déshabillé honnête d'une bourgeoise dans la maison de son père à la campagne.

CORNALINE, costume de soubrette.

LARABESQUE, juif anglais: perruque rousse, la petite barbe de même, frac anglais, gilet, culotte de peau, brodequins, chapeau rond.

CAPITOLIN doit être entièrement vêtu de bandes de feuilles d'impression. Il faut qu'elles soient cousues sur son habit, veste;-culotte et bas, et que la totalité de son vêtement en soit couvert, il faut absolument se garder de mettre des feuilles manuscrit le contresens serait évident, il faut absolument des feuilles d'impression: il n'aura jamais de chapeau sur sa tête...

DELCOUR aura un petit frac anglais assez mesquin; un chapeau rond».

(91) Henriette et Saint-Clair ou La Force du sang, t. 4, p. 443; «Costumes.

M. de VOLSANGE vêtu en bon propriétaire de campagne.

SAINT-CLAIR d'abord en jeune homme qui revient de la chasse, ensuite en officier des dragons.

VALVILLE en habit de campagne simple.

LE VOYAGEUR le costume de la misère mais non

pas sous des habits déguenillés.

PAULINE le costume bourgeois le plus simple.

HENRIETTE le négligé le plus modeste.

$\mathrm{M}^{\mathrm{ME}}$ de LORMEUIL l'habit sans luxe d'une femme

qui est à la campagne chez elle.

LAPINEAU en garde-chasse».

LUCETTE en paysanne. 
reçoit, pour la première fois, dans sa demeure, notre malheureuse héroïne ${ }^{92}$, par exemple.

La relative négligence à l'égard de la chevelure n'est pas inconvenante dans la vie privée des individus de l'aristocratie et du haut clergé, voire de la haute bourgeoisie, sous l'Ancien Régime. Aussi, il n'est pas surprenant de rencontrer certains personnages ayant les «cheveux flottant en boucles sur leurs épaules» ${ }^{93}$ ou bien encore, à l'instar de Léonore qui, au château de Vertfeuille, chez madame de Blamont, offre au lecteur de «très beaux cheveux noirs» «négligemment attachés» ${ }^{94}$. Enfin, dans Les Cent vingt journées de Sodome, le narrateur nous prête à voir «quatre jeunes amants» dont les «cheveux négligemment relevés de quelques boucles sur les côtés» sont «absolument libres et flottants par-derrière» ${ }^{95}$. En revanche dans Justine ou les Malheurs de la vertu, cette négligence prend un autre sens. Lorsque le narrateur révèle au lecteur-spectateur que Juliette a «les cheveux traînants» ${ }^{96}$ au moment de son départ du couvent et de son entrée dans le monde, après le décès de ses parents, le manque d'attention apporté à sa coiffure, -détail anodin en apparence-, préfigure bien le désordre physique et moral qui habitera notre Messaline tout au long de sa vie, dans la mesure où le soin accordé à la chevelure ou le port d'une perruque fait partie intégrante du savoir-vivre, des convenances et de la bienséance qui marquent les relations entre les individus, dans la société policée du XVIII ${ }^{\text {ème }}$ siècle.

Nous avons pu constater enfin que les coiffures servent aussi à regrouper en différentes classes les victimes nécessaires aux débauches des libertins. Dans La Nouvelle Justine..., les jeunes femmes retenues au couvent de Sainte-Marie-des-Bois reçoivent un «modèle» de coiffure qui leur est imposé et qui change régulièrement en fonction de la classe à laquelle elles appartiennent:

Nous sommes obligées de nous coiffer nous-mêmes, ou mutuellement. Les modèles nous sont donnés; ils varient tous les deux mois; chaque classe a son modèle à part ${ }^{97}$.

Même souci de classification dans l'Histoire de Juliette..., puisque le duc de Pienza exige, pour ses plaisirs lubriques, «seize jolies filles» que Sbrigani et Juliette doivent «arranger par couples», en prenant soin de «caractériser chaque couple» d'«une coiffure égale» ${ }^{98}$. Quant aux hommes de Tamoé, dans Aline et Valcour, ceuxci ont tous

la tête couverte d'une espèce de turban léger d'une forme très agréable, et de la même couleur que leur vêtement ${ }^{99}$.

Nous le constatons, le souci du détail vestimentaire n'est pas un vain mot dans le romans sadien. S'il est indéniable que Donatien y attache tant d'importance pour des raisons de réalisme et de vraisemblance, il n'en demeure pas moins vrai qu'une autre explication à ce phénomène est parfaitement envisageable. Le costume, dans l'ensemble des cas que nous avons pu étudier, sert essentiellement à embellir le corps, à le mettre «sur son trente-et-un», si l'on peut dire, avant que ce corps ne soit totalement

(92) Justine ou les Malbeurs de la vertu, t. 3, p. 197; La Nouvelle Justine ou les Malbeurs de la vertu, t. 7 , p. 118.

(93) Histoire de Juliette ou les Prospérités du vice, t. 9, p. 202.

(94) Aline et Valcour, t. 4, p. 178. 145.

(95) Les Cent vingt journées de Sodome, t. 1, p.
(96) Justine ou les Malheurs de la vertu, t. 3, p. 29.

(97) La Nouvelle Justine ou les Malheurs de la vertu, t. 6, p. 299.

(98) Histoire de Juliette ou les Prospérités du vice, t. 9 , p. 31 .

(99) Aline et Valcour, t. 4, p. 283. 
dénudé et réduit à l'état d'objet entre les mains du libertin. Madame de Gernande, avant de subir les brimades de son époux sanguinaire, lui est présentée «simplement entourée d'une robe de mousseline flottante». Et Justine devra, sur l'ordre du comte, «déshabiller sa femme et la lui conduire» afin de soumettre la pauvre comtesse au supplice de la saignée ${ }^{100}$; le même sort est réservé à la malheureuse Sophie dans Les Cent vingt journées de Sodome. Cette dernière est présentée au duc de Blangis dans un «accoutrement de novice» qui produit un tel effet sur notre roué que ce dernier désire ardemment «lui barbouiller le con de foutre» après avoir sommé «qu'on la déshabille» ${ }^{101}$.

Il arrive aussi que Donatien, à la manière d'un auteur dramatique qui distribue les rôles et précise la valeur symbolique des costumes portés par chacun des acteurs entrant en scène, affuble de tenues diverses tous les personnages qui participent à une scène orgiaque et détaille chacune de celles-ci avant qu'elles ne soient abandonnées. C'est ce que nous constatons, par exemple, dans La Nouvelle Justine...:

À dix heures précises du matin, la société se rendit au local préparé, et chacun vêtu d'un costume différent, que nous allons tracer en nommant chaque acteur.

$\mathrm{M}^{\mathrm{me}}$ de Verneuil y parut vêtue à la manière des sultanes de Constantinople. Aucune parure sans doute n'eût autant servi sa beauté.

Cécile, sa charmante fille, était en brun, sous le costume exact des marmottes de la vallée de Barcelonnette. On n'imagine pas les désirs qu'elle inspirait sous cet habit [...].

Marceline était en sauvage.

Sa jeune fille Laurette s'y voyait sous une simple simarre de gaze écrue, agréablement renouée sur les hanches et sur le sein gauche avec de gros flots de ruban lilas; un des tétons et la moitié de ses fesses s'apercevaient par ce moyen [...].

$\mathrm{M}^{\mathrm{me}}$ de Gernande y vint sous le costume intéressant des victimes qu'on immolait au temple de Diane; on l'eût prise pour Iphigénie.

Justine était en soubrette, les bras nus; agréablement couronnée de roses, et sa jolie taille bien développée $[\ldots .$. .

Sous celui d'Hercule et de Mars, parurent John et Constant, les deux valets de chambre de Verneuil.

Lui, d'Esterval, Bressac et Gernande s'y montrèrent revêtus de pantalons de soie rouge, qui leur collaient exactement sur la peau et qui les enfermaient scrupuleusement depuis la nuque du cou jusqu'aux pieds. Une ouverture ronde, artistement pratiqués par-devant et parderrière, laissait à nu les fesses et leurs vits. Ils avaient beaucoup de rouge, et sur la tête un léger turban ponceau. Ils ressemblaient à des furies ${ }^{102}$.

Mais il apparaît aussi que le costume peut être considéré comme un vecteur d'humanisation de la victime ou du libertin en devenir. Le vêtement prend alors valeur d'unique trait d'union, de passerelle entre le monde policé et régi par des lois morales de toute nature et le monde libertin dépourvu de tout code éthique. On perçoit très bien ce phénomène dans La Philosophie dans le boudoir au moment de l'entrée en scène d'Eugénie. Après qu'elle a accueilli le plus civilement du monde Eugénie, dans le deuxième dialogue, madame de Saint-Ange, dans le troisième, ne perd pas une minute pour se lancer dans l'éducation de notre jeune ingénue et commence par la «déshabiller». En abandonnant ses vêtements, Eugénie abandonne le monde des bienséances et des codes moraux, de sorte que la nudité qui en résulte la plonge irrémédiablement dans le monde du libertinage ${ }^{103}$. On peut établir le même

(100) Justine ou les Malheurs de la vertu, t. 3, pp. 208-209.

(101) Les Cent vingt journées de Sodome, t. 1, p. 119.
(102) La Nouvelle Justine au les Malbeurs de la vertu, t.7, pp. 181-182.

(103) La Philosophie dans le boudoir, t. 3, p. 
constat lors de la mise en scène du pseudo-miracle de Sainte-Marie-des-Bois. La jeune Florette qui est parée des habits de la vierge, - symbole s'il en est d'un monde empreint de moralité et de respect d'autrui -, doit paraître ainsi vêtue aux orgies du soir avant d'être mise à nu et victime des pires déportements ${ }^{104}$. Enfin, dans l'Histoire de Juliette..., Dorval, dans un geste pouvant signifier le refus d'admettre que le vêtement est facteur d'humanisation et du respect d'autrui, jette, «par une fenêtre» ${ }^{105}$, les «hardes» de ses victimes, après les avoir mises à nu.

En dernier lieu, les costumes que portent certains personnages permettent de regrouper ces derniers en différentes classes en fonction du rôle qu'ils ont à jouer dans telle ou telle bacchanale. Les costumes, dès lors, ne sont plus de simples vêtements servant à embellir le corps, mais deviennent proprement des accessoires rituels nécessaires au culte du Libertinage, au même titre que les ornements sacerdotaux sont indispensables pour un prêtre lors d'un office religieux. C'est ce que nous retrouvons dans La Nouvelle Justine... ${ }^{106}$ et dans Justine ou les Malheurs de la vertu.

Toutes les pensionnaires de Sainte-Marie-des-Bois sont regroupées dans «quatre classes» différentes en fonction de leur âge. Et chacune des classes est reconnaissable à la couleur du costume que portent ses pensionnaires. Les jeunes filles de la «classe de l'enfance» portent «un habillement blanc», celles de «la classe de la jeunesse», sont vêtues de «vert», «la troisième classe», «celle de l'âge raisonnable» s'habillent de vêtements «bleus», et les membres de la dernière classe «destinée pour l'âge mûr», sont «vêtue[s] de mordoré» ${ }^{107}$. Dans Aline et Valcour, Sainville apprend de Zamé que la population de Tamoé, en fonction de l'âge de ses membres, est répartie en trois classes dont chacune porte des habits gris ou verts ou rose ${ }^{108}$. Dans Les Cent vingt journées de Sodome, enfin, le costume peut se limiter à un simple ruban dont la couleur est choisie par l'un de nos quatre roués de sorte qu'il devient facile de savoir de qui dépend telle ou telle victime lorsque l'heure des orgies sera venue:

Les amis étant bien aises de distinguer à tout instant de la journée ceux des jeunes gens, soit en filles, soit en garçons, dont les pucelage devaient leur appartenir, décidèrent de leur faire porter, dans tous leurs divers ajustements, un ruban à leurs cheveux qui indiquât à qui ils appartenaient En conséquence, le duc adopta le rose et le vert, et tout ce qui aurait un ruban rose par-devant lui appartiendrait pour le con, de même que tout ce qui en porterait un vert par-derrière serait à lui pour le cul [...]. Curval prit le noir pour le devant et le jaune pour le derrière, [...]. Durcet marqua le seul Hyacinthe d'un ruban lilas par-derrière, et l'évêque, qui n'avait pour lui que cinq pré-mices sodomites, ordonna à Cupidon, Narcisse, Céladon, Colombe et Fanny d'en porter un violet par-derrière ${ }^{109}$.

avant que ces rubans ne deviennent les signes distinctifs d'un supplice particulier qu'on afflige à de malheureuses jeunes filles, dans les dernières pages de L'École $d u$ libertinage:

Mais avant de les [quinze filles] précipiter ainsi, il leur passe un ruban au col, et ce ruban qui signifie un supplice est analogue à celui auquel il s'imagine qu'elles seront le plus propres, ou qui deviendra le plus voluptueux à leur infliger. [...] Le caveau où les filles tombent est garni de quinze différents assortiments de supplices effroyables, et un bourreau, sous le masque et l'emblème d'un démon, préside à chaque supplice, vêtu de la couleur affectée à

(104) Justine ou les Malheurs de la vertu, t. 3, p. 181.

(105) Histoire de Juliette ou les Prospérités du vice, t. 8, p. 166.

(106) La Nouvelle Justine ou les Malheurs de la vertu, t. 6, p. 258.
(107) Justine ou les Malbeurs de la vertu, t.3, p. 145.

(108) Aline et Valcour, t. 4, p. 283.

(109) Les Cent vingt journées de Sodome, t. 3 , p. 135. 
ce supplice. Le ruban que la fille a au col répond à une des couleurs affectées à ces supplices, et dès qu'elle tombe, le bourreau de cette couleur s'empare d'elle et la mène au supplice où il préside $[\ldots]^{110}$.

Si les décors intérieurs et les costumes ont une importance relative dans la mise en scène du monde romanesque sadien, l'un et l'autre de ces deux aspects n'en revêtent pas moins une importance capitale puisqu'ils participent, chacun à sa manière, à la mise en scène des personnages et des corps cher au divin marquis.

DIDIER DERSON 